\title{
Volumetric body composition parameters in predicting survival outcomes of metastatic renal cell carcinoma patients treated with targeted therapy
}

\author{
Oktay Aktepe ${ }^{1}$, Ahmet Gürkan Erdemir², Gurkan Guner ${ }^{3}$, Deniz Can Güven ${ }^{3}$, Suayib \\ Yalcin $^{3}$, Mehmet Ruhi Onur ${ }^{3}$, and Mustafa Erman ${ }^{3}$ \\ ${ }^{1}$ Affiliation not available \\ ${ }^{2}$ Hacettepe University Faculty of Medicine \\ ${ }^{3}$ Hacettepe University
}

May 1, 2021

\begin{abstract}
Background: To explore the clinical significance of baseline volumetric body composition parameters evaluated with computerized tomography $(\mathrm{CT})$ and their changes after 3-4 months from treatment initiation of targeted therapy in patients with metastatic renal cell carcinoma (mRCC). Method: This study included 108 Caucasian mRCC patients (Male/Female: 77/31) treated with targeted therapy. Volumetric body composition parameters including total adipose tissue index (TATI), subcutaneous adipose tissue index (SATI), visceral adipose tissue index (VATI) and skeletal muscle index (SMI) values were depicted from CT images at third lumbar vertebra level through volumetric measurement software. Kaplan-Meier method and the long test were used for estimation of progression free survival (PFS) and overall survival (OS). Univariate and multivariate analyses were done to determine the associations between clinic-pathologic variables including VBC and survival outcomes. Results: The median PFS and OS of all patients were 11 months and 46 months in patients respectively. After adjustment for the variables including international mRCC database consortium (IMDC) risk score, only a high skeletal muscle index (SMI) was associated with better PFS (HR: 0.975, $\mathrm{P}=0.015)$. The independent predictors for OS were VATI (HR 1.005, P=0.024), SATI (HR: 0.976, $\mathrm{P}=0.019$ ) and TATI (HR: 0.982, $\mathrm{P}=0.035$ ) in addition to IMDC risk score. Conclusion: Our findings revealed that while SMI was the only significant determinant parameter for PFS among VBC parameters, TATI, VATI, and SATI were determined as independent predictors for OS in addition to IMDC risk score.
\end{abstract}

Volumetric body composition parameters in predicting survival outcomes of metastatic renal cell carcinoma patients treated with targeted therapy

Oktay Halit Aktepe ${ }^{1, *}$, Ahmet Gurkan Erdemir ${ }^{2}$, Gurkan Guner ${ }^{3}$, Deniz Can Guven ${ }^{3}$, Suayib Yalcin ${ }^{3}$, Mehmet Ruhi Onur ${ }^{2}$, Mustafa Erman ${ }^{4}$

Running title: Volumetric body composition in metastatic renal cell carcinoma

\section{Affiliations:}

${ }^{1}$ Department of Medical Oncology, Bolu Abant Izzet Baysal University, 14030, Bolu, Turkey.

${ }^{2}$ Department of Radiology, Hacettepe University, 06100, Sihhiye, Ankara, Turkey

${ }^{3}$ Department of Medical Oncology, Hacettepe University, 06100, Sihhiye, Ankara, Turkey.

${ }^{4}$ Department of Preventive Oncology, Hacettepe University, 06100, Sihhiye, Ankara, Turkey 


\section{*Corresponding Author:}

ORCID ID: 0000-0003-3540-0701

Oktay Halit Aktepe, M.D.

Bolu Abant Izzet Baysal University, Faculty of Medicine

Department of Internal Medicine, Division of Medical Oncology

14030, Bolu, Turkey

Phone: +90-554-114-65-99

Fax: +90-374-270-00-66

E-mail: droktayaktepe@hotmail.com

\section{Acknowledgment}

None.

\section{Abstract}

Background: To explore the clinical significance of baseline volumetric body composition parameters evaluated with computerized tomography (CT) and their changes after 3-4 months from treatment initiation of targeted therapy in patients with metastatic renal cell carcinoma (mRCC).

Method: This study included 108 Caucasian mRCC patients (Male/Female: 77/31) treated with targeted therapy. Volumetric body composition parameters including total adipose tissue index (TATI), subcutaneous adipose tissue index (SATI), visceral adipose tissue index (VATI) and skeletal muscle index (SMI) values were depicted from CT images at third lumbar vertebra level through volumetric measurement software. Kaplan-Meier method and the long test were used for estimation of progression free survival (PFS) and overall survival (OS). Univariate and multivariate analyses were done to determine the associations between clinic-pathologic variables including VBC and survival outcomes.

Results: The median PFS and OS of all patients were 11 months and 46 months in patients respectively. After adjustment for the variables including international $\mathrm{mRCC}$ database consortium (IMDC) risk score, only a high skeletal muscle index (SMI) was associated with better PFS (HR: 0.975, $\mathrm{P}=0.015)$. The independent predictors for OS were VATI (HR 1.005, $\mathrm{P}=0.024$ ), SATI (HR: 0.976, $\mathrm{P}=0.019$ ) and TATI (HR: $0.982, \mathrm{P}=0.035)$ in addition to IMDC risk score.

Conclusion: Our findings revealed that while SMI was the only significant determinant parameter for PFS among VBC parameters, TATI, VATI, and SATI were determined as independent predictors for OS in addition to IMDC risk score.

Keywords: Metastatic renal cell carcinoma; obesity; volumetric body composition; prognosis

\section{What is already known about this topic?}

Obesity is a well-known risk factor for development of renal cell carcinoma. However, the clinical importance of obesity and volumetric body composition parameters (VBC), visceral adipose tissue (VATI), subcutaneous adipose tissue index (SATI), in predicting survival outcomes of metastatic renal cell carcinoma (mRCC) treated with targeted therapy, sunitinib or pazopanib, remain to be elucidated. The present study aimed to investigate the prognostic value of both VBC parameters and skeletal muscle index, an indicator of sarcopenia, in mRCC patients treated with targeted therapy.

\section{What does this article add?}


The independent predictors of overall survival were SATI and VATI, whereas only SMI was determined as independent factor in predicting progression free survival in mRCC treated with targeted therapy. We for the first time evaluated a wide range of VBC parameters and sarcopenia indicators in one cohort.

\section{Introduction}

Renal cell carcinoma (RCC) constituting $4 \%$ of all cancers is the sixth and the tenth most frequently diagnosed cancer type in men and women worldwide, respectively [1]. Although most of the lesions detected incidentally via developed imaging techniques were small and low-grade tumors, $17 \%$ of all RCC cases present with distant metastasis at diagnosis [2]. Due to recent advances, the first-line treatment for metastatic renal cell carcinoma $(\mathrm{mRCC})$ has evolved from cytokine-based treatment to vascular endothelial growth factor (VEGF) pathway inhibitors, immune checkpoint blockers, and their combination. To define the prognosis of mRCC patients, two models named "International Metastatic Renal Cell Carcinoma Database Consortium (IMDC)" and "The Memorial Sloan-Kettering Cancer Center (MSKCC) Criteria" were established, and mRCC patients were classified in terms of risk levels as favorable, intermediate and poor $[3,4]$.

Although obesity is linked to increased risk of developing RCC, underlying mechanisms of this link is poorly understood [5]. It has been demonstrated that the biological mechanisms of obesity leads to development of RCC including chronic tissue hypoxia, change of the hormonal environment in the fat tissue, and immune dysfunction [6,7]. Interestingly, some studies showed that obese $\mathrm{mRCC}$ patients have a better response to treatment and thereby a better prognosis as it has been named as the 'obesity paradox' in the literature $[8,9]$. This 'risk factor' paradox may be due to the time gap between two detrimental risk factors that overnutrition is a well known long-term killer but can be a protective factor in the short term, while malnutrition is a short-term killer [10]. Additionally, the decline of skeletal muscle, called sarcopenia, has been reported as a predictor of prognosis in various tumors including RCC [11-13]. Also, it has been shown that the decline in skeletal muscle mass is related to increased side effects in cancer treatment and worsened survival outcomes $[14,15]$.

Body Mass Index (BMI) is a rough sketch of the distribution of fat in the body. Hence, BMI alone is not sufficient to detect the exact body composition parameters and the sarcopenia, which is characterized by the loss of body fat composition and skeletal muscle tissue. Computerized tomography (CT) is routinely used to determine staging and treatment response evaluation of RCC. This technique allows for the better categorization of fat and skeletal muscle tissue. Additionally, CT can be used in the relative classification of the fat tissue in visceral and subcutaneous compartments [visceral adipose tissue (VAT) and superficial adipose tissue (SAT)]. The studies on the effects of these parameters on the prognosis of mRCC delivered different results. Steffens et al. [8] and Gu et al. [16] demonstrated that the higher VAT and SAT were correlated with prolonged OS in mRCC patients. Contrary to these findings, increased VAT was found to be correlated with poor outcomes in the Ladoire's cohort [17]. A study by Antoun et al. demoonstrated no correlation between VAT or SAT and survival outcomes in patients with mRCC [18]. It has been shown that body weight and tissue loss can contribute to predicting the prognosis of mRCC patients treated with targeted therapy [19]. However, few studies have evaluated the prognostic value of longitudinal changes in parameters of body composition over time in mRCC patients treated with targeted therapy. The current study aimed to investigate whether or not obesity and volumetric body composition (VBC) parameters can predict the outcomes in mRCC patients treated with targeted therapy (pazopanib or sunitinib) in terms of VAT, subcutaneous SAT, BMI, skeletal muscle area (SMA) measurements and their changes after 3-4 months after initial CT.

\section{Materials and methods}

\subsection{Patients}

The study population comprised 108 Caucasian patients with confirmed mRCC and aged 18 years old or older being treated with anti-angiogenic therapy from January 2008 to May 2019 at Hacettepe University Cancer Institute (Ankara, Turkey). The baseline demographic, clinical, and laboratory data which were previously documented to have prognostic value were obtained from our center's electronic database [4]. Patients only 
with histologically proven $\mathrm{mRCC}$ and good performance statues (Karnofsky performance status [?]80) were included in this study. Patients who lost to follow-up and with no abdominal CT scan data at either before treatment initiation or after 3-4 months of targeted VEGF therapy were excluded from the study. The study approved by the local ethics committee and written informed consent were obtained from all of the research participants or their relatives. All procedures in the study were performed in accordance with the 1964 Helsinki declaration and its later amendments.

\subsection{Imaging analysis}

Radiological measurements were performed by two radiologists in cooperation (RO, AGE), who were blinded to all clinical information of patients. Contrast-enhanced (CT) images of all patients with $5 \mathrm{~mm}$ section thickness at the venous phase were selected. For the interpretation of each CT image, axial CT images oriented parallel to superior and inferior endplates of L3 vertebrae were selected. The CT images were evaluated via Region Growing application of Syngo.Via Software (Siemens Healthcare GmbH, Germany). Within every cross-sectional CT image, SMA, total adipose tissue (TAT), SAT and VAT were determined separately and outlined semi-automatically. These parameters were standadized according to the stature and represented in units of $\mathrm{cm}^{2} / \mathrm{m}^{2}$ [VAT index (VATI), superficial adipose tissue (SATI), TAT index (TATI), and SMA index (SMI)], as described previously [19].

\subsection{Statistical Analysis}

The descriptive analyses were presented as median and mean with standard deviation for numerical data, and frequency and and percent for categorical data. The comparisons between independent groups were performed using Mann-Whitney U and Chi-square for numerical and categorical variables, respectively. The overall survival (OS) and progression-free survival (PFS) were calculated as the time frame from diagnosis to last follow up and/or death for OS, and time frame from treatment initiation to progression and/or death for PFS. The median follow-up was calculated as the time from diagnosis to last follow-up or death date. The survival comparisons were done using the Kaplan-Meier method and the log-rank test. The variables with a p-value $<=0.25$ were considered as candidate variables for multivariate analyses. SPSS 25 software (IBM Inc., Armonk, NY, USA) was used for the analyses in the study, and a p-value below 0.05 was considered as statistically significant.

\section{Results}

\subsection{Baseline patient characteristics and study design}

Baseline patient and tumor characteristics of $108 \mathrm{mRCC}$ patients are presented according to gender in Table 1. The whole study population received targeted therapy (pazopanib, $n=64$; sunitinib, $n=44$ ). Clear cell histology $(72.2 \%)$ was the most common subtype. During a median follow-up of 22 months (range: $2-102$ months), $38(35.2 \%)$ patients died and $28(25.9 \%)$ patients were considered obese (BMI of [?]30 $\left.\mathrm{kg} / \mathrm{m}^{2}\right)$. According to the IMDC criteria [4]; of the 108 patients, 9 (8.3\%) were in favorable-risk group, $66(61.1 \%)$ in intermediate-risk group and $33(30.5 \%)$ in poor-risk group.

Body composition parameters differed between men and women listed in Table 2. While median baseline total adipose tissue index (TATI) and subcutaneous adipose tissue index (SATI) were significantly higher in females than males, median baseline skeletal muscle index $(\mathrm{SMI})$ was higher in males $(\mathrm{P}<0.001)$. None of the second and change measures of all VBC parameters exhibited a significant difference among males and females and also none of the VBC parameters was associated with pathologic features of RCC including histological subtype, tumor grade, or tumor size (data not shown)

\subsection{The effect of body composition parameters on survival outcomes}

The measured median PFS and OS times of all 108 patients with metastatic RCC were 11 (95\% CI: 8-14) months and 46 (95\% CI: 42.9-49.1) months, respectively. Among the obese (BMI[?]30 kg/m²) and non-obese $\left(\mathrm{BMI}<30 \mathrm{~kg} / \mathrm{m}^{2}\right)$ patients, Kaplan-Meier analysis revealed a significant difference in the PFS (median 14 and 8 months, respectively, $\mathrm{P}=0,039$ ) while no significant difference was found in OS (median, 46 and 47 months, 
respectively, $\mathrm{P}=0,921)$. The median PFS times for each IMDC risk groups were 21 months (favorable-risk group; 95\% CI: 16.8-25.2), 14.0 months (intermediate-risk group; 95\% CI: 11.9-16.1) and 6 months (poor-risk group; 95\% CI 4.9-7.1). The median OS times for each risk group were 48 months (favorable-risk group; 95\% CI: 46-50), and 46 months (intermediate-risk group; $95 \%$ CI: 43-49) and 28 months (poor risk; $95 \%$ CI: $25-32)$.

Using univariate Cox regression analyses as presented in Table 3, none of the body composition variables and their changes after 3-4 months of treatment initiation were associated with OS while SMI (HR: 0.979, 95\% CI: 0.961-0.997, $\mathrm{P}=0.021$ ) and psoas index (HR: 0.871, 95\% CI: 0.776-0.978, $\mathrm{P}=0.020$ ) were identified as the factors predicting better PFS. Multivariate Cox regression models have identified the independent prognostic factors for PFS and OS (Table 4). When adjusted by significant and potential variables in univariate analyses, only SMI was determined as an independent prognostic factor for PFS (HR: 0.975; 95\% CI: $0.955-0.995, \mathrm{P}=0.015)$. However, SMI was not found as a significant factor for OS. Although we found no association between VBC parameters or change in their values and OS according to univariate analyses; applying multivariate analyses, VATI (HR: 1.005; 95\% CI: 1.001-1.009, P=0.024), SATI (HR: 0.976, 95\% CI: 0.957-0.996, $\mathrm{P}=0.019$ ) and TATI (HR: 0.982, 95\% CI: 0.966-0.999, $\mathrm{P}=0.035)$ were determined as independent predictors for OS in addition to IMDC scoring system.

\section{Discussion}

The development of VEGF pathway blocking agents led to a significant breakthrough in the outcome of mRCC patients. Besides, immune checkpoint inhibitors are used either alone or in combination with VEGF blocking agents for the treatment $\mathrm{mRCC}$ patients [20,21]. With the availability of alternative treatment options, novel prognostic markers are of paramount importance to provide better personalized treatments. Current prognostic scoring systems have been developed based on clinical and laboratory parameter values $[3,4]$. Obesity is not included among these values, and its impact on the prognosis of mRCC patients is still controversial [22]. For this reason, in addition to BMI; VBC measurements, suggested as more reliable indicators of obesity, have been investigated in our study. In the present study, the impact of baseline VBC and sarcopenia parameters as well as their post-treatment changes at the third-fourth month in the mRCC patients treated with targeted therapy were analyzed. To the best of our knowledge, we for the first time analyzed the associations of several VBC parameters and their changes with PFS and OS in one cohort. Although obesity is a well-defined risk factor for RCC; as a paradox to this situation, we found that TATI, SATI, and VATI were independent predictive factors for OS.

Body composition may be defined as the amount and distribution of lean tissue, fat tissue, and bones. Up to this decade, studies on body composition and clinical outcomes of mRCC have been mainly investigated with BMI values. However, the exact amount of fat tissue or rate of fat to muscle tissues could not be predicted from BMI values alone. With advances in imaging technologies, specific analysis of muscle and fat tissue may be obtained from CT taken for the staging of oncologic diseases, and response assessment purposes. Thus, in addition to BMI, the studies focused on the measurement of SAT and VAT components of the intra-abdominal fat tissue at the umbilicus-level using CT. Steffens et al. conducted a study focusing on the impact of VAT and SAT on the prognosis of $116 \mathrm{mRCC}$ patients and found that contrary to the BMI, above average values for VFA and SFA are related to longer PFS and OS in mRCC patients (SAT; HR: 3.41, 95\% CI: 1.61-7.25, $\mathrm{P}=0.001$; VAT; HR: 2.97, 95\% CI: 1.36-6.47, $\mathrm{P}=0.006$ ) [8]. Ladoire et al. assessed the prognostic value of SAT, VAT, and BMI in $64 \mathrm{mRCC}$ patients treated with targeted therapy using same measurement techniques as in the Steffens' trial [17]. However, they reported that high VAT was associated with significantly worse OS (HR: 6.26, 95\% CI: 2.29-17.08, P<0.001) [17]. The contradicting results in these studies may have been due to cut-off points not defined per gender and/or CT measurements of VBC through umbilicus level. It would be incorrect to evaluate the CT measurements taken at this level to estimate the full-body fat and muscle tissues. Hence, VAT and SAT were not associated with prognosis of $\mathrm{mRCC}$ in a study by Antoun et al. when these measurements were performed on a more appropriate level as L3 and adjusted per gender [19]. Additionally, these studies were performed with baseline measurements. Based on these results, VBC were measured with at the L3 level and adjusted according to gender in the 
present study and we also evaluated the effect of CT measurement changes (the value between the initial CT and the CT performed 3-4 months after treatment initiation) on PFS and OS.

Nevertheless, the present study had several limitations. Firstly, the study population was relatively small and consisted of single-center patients. Secondly, the present study had a retrospective design and the relatively short median follow-up time. Furthermore, because most patients in our cohort were categorized as an intermediate-risk group (61\%), our results should be further investigated in favorable and poor risk groups in a more homogeneous population. From this perspective, these disparate results in the literature mentioned above may emerge from an disparity in prognostic factors not regulated in one study than in another.

\section{Conclusion}

In summary, our findings suggest that high levels of adipose tissue in CT parameters including TATI, VATI, and SATI may be a possible predictor of OS in patients with mRCC treated with targeted therapy, and also SMI may be a predictor for PFS. Considering the widely disparate results of the studies, the prognostic value of body composition (BMI, SATI, VATI, SMI) and their alterations in the course of mRCC should be evaluated in a larger patient population with prospective design to validate cut-off values for these parameters. If our results are corroborated by future trials, the measurement of VBC parameters and incorporation of those into the current scoring systems would provide better prognostication and personalized treatment in $\mathrm{mRCC}$.

\section{Conflict of interest}

The authors declared that they had no conflicts of interests.

\section{Funding}

This study did not receive funding.

\section{Author Contributions}

OHA, MRO, and ME designed research; OHA, AGE, DCG and GG conducted research; OHA, MRO, and ME analyzed data; OHA, AGE, MRO, SY and ME wrote the paper; OHA had primary responsibility for final content. All authors read and approved the final manuscript.

\section{References}

1. Siegel RL, Miller KD, Jemal A. Cancer statistics, 2016. CA Cancer J Clin 2016;66:7-30.

2. Capitanio U, Montorsi F. Renal cancer. Lancet 2016;387:894-906.

3. Motzer RJ, Bacik J, Mazumdar M. Prognostic factors for survival of patients with stage IV renal cell carcinoma: memorial sloan-kettering cancer center experience. Clin Cancer Res 2004;10:6302s-6303s.

4. Heng DY, Xie W, Regan MM, et al. Prognostic factors for overall survival in patients with metastatic renal cell carcinoma treated with vascular endothelial growth factor-targeted agents: results from a large, multicenter study. J Clin Oncol 2009;27:5794-5799.

5. Klinghoffer Z, Yang B, Kapoor A, Pinthus JH. Obesity and renal cell carcinoma: epidemiology, underlying mechanisms and management considerations. Expert Rev Anticancer Ther 2009;9:975-987.

6. Calle EE, Kaaks R. Overweight, obesity and cancer: epidemiological evidence and proposed mechanisms. Nat Rev Cancer 2004;4:579-591.

7. Donat SM, Salzhauer EW, Mitra N, Yanke BV, Snyder ME, Russo P. Impact of body mass index on survival of patients with surgically treated renal cell carcinoma. J Urol 2006;175:46-52. 
8. Steffens S, Grünwald V, Ringe KI, et al. Does obesity influence the prognosis of metastatic renal cell carcinoma in patients treated with vascular endothelial growth factor-targeted therapy? Oncologist 2011;16:15651571.

9. Hakimi AA, Furberg H, Zabor EC, et al. An epidemiologic and genomic investigation into the obesity paradox in renal cell carcinoma. J Natl Cancer Inst 2013;105:1862-1870.

10. Kalantar-Zadeh K, Horwich TB, Oreopoulos A, et al. Risk factor paradox in wasting diseases. Curr Opin Clin Nutr Metab Care 2007;10:433-442.

11. Harimoto N, Shirabe K, Yamashita YI, et al. Sarcopenia as a predictor of prognosis in patients following hepatectomy for hepatocellular carcinoma. Br J Surg 2013;100:1523-1530.

12. Miyamoto Y, Baba Y, Sakamoto Y, et al. Sarcopenia is a Negative Prognostic Factor After Curative Resection of Colorectal Cancer. Ann Surg Oncol 2015;22:2663-2668.

13. Gu W, Wu J, Liu X, et al. Early skeletal muscle loss during target therapy is a prognostic biomarker in metastatic renal cell carcinoma patients. Sci Rep 2017;7:7587.

14. Prado CM, Lieffers JR, McCargar LJ, et al. Prevalence and clinical implications of sarcopenic obesity in patients with solid tumours of the respiratory and gastrointestinal tracts: a population-based study. Lancet Oncol 2008;9:629-635.

15. Antoun S, Baracos VE, Birdsell L, Escudier B, Sawyer MB. Low body mass index and sarcopenia associated with dose-limiting toxicity of sorafenib in patients with renal cell carcinoma. Ann Oncol 2010;21:15941598 .

16. Gu W, Zhu Y, Wang H, et al. Prognostic value of components of body composition in patients treated with targeted therapy for advanced renal cell carcinoma: a retrospective case series. PLoS One 2015;10:e118022.

17. Ladoire S, Bonnetain F, Gauthier M, et al. Visceral fat area as a new independent predictive factor of survival in patients with metastatic renal cell carcinoma treated with antiangiogenic agents. Oncologist 2011;16:71-81.

18. Antoun S, Lanoy E, Iacovelli R, et al. Skeletal muscle density predicts prognosis in patients with metastatic renal cell carcinoma treated with targeted therapies. Cancer 2013;119:3377-3384.

19. Antoun S, Birdsell L, Sawyer MB, Venner P, Escudier B, Baracos VE. Association of skeletal muscle wasting with treatment with sorafenib in patients with advanced renal cell carcinoma: results from a placebocontrolled study. J Clin Oncol 2010;28:1054-1060.

20. Powles T, Albiges L, Staehler M, et al. Updated European Association of Urology Guidelines: Recommendations for the Treatment of First-line Metastatic Clear Cell Renal Cancer. Eur Urol 2018;73:311-315.

21. Motzer RJ, Penkov K, Haanen J, et al. Avelumab plus Axitinib versus Sunitinib for Advanced Renal-Cell Carcinoma. N Engl J Med 2019;380:1103-1115.

22. Yip SM, Heng DY, Tang PA. Review of the Interaction Between Body Composition and Clinical Outcomes in Metastatic Renal Cell Cancer Treated With Targeted Therapies. J Kidney Cancer VHL 2016;3:12-22.

Table 1. Clinical-pathological characteristics of patients stratified according to gender.

\begin{tabular}{lllll}
\hline Characteristics & $\begin{array}{l}\text { All patients }(\mathrm{n}= \\
108)\end{array}$ & Female $(\mathrm{n}=31)$ & Male (n=77) & P value \\
\hline Age,years & $57(53-66)$ & $56(52-64)$ & $57(55-68)$ & 0.659 \\
Histology Clear cell & $78(72.2 \%) 30(27.8$ & $27(87 \%) 4(13 \%)$ & $57(74 \%) 20(26 \%)$ & 0.734 \\
Non-clear cell & $\%)$ & & & \\
Metastatic region & $88(81.5 \%) 20$ & $24(81.5 \%) 7$ & $64(81.5 \%) 13$ & 0.490 \\
sites 1-2 3-4-5 & $(18.5 \%)$ & $(18.5 \%)$ & $(18.5 \%)$ &
\end{tabular}




\begin{tabular}{lllll}
\hline Characteristics & All patients $(\mathrm{n}=$ & Male $(\mathrm{n}=77)$ & P value \\
\hline Tumor Grade I II & $5(4.6 \%) 20(18.6 \%)$ & $3(4 \%) 5(17.8 \%) 11$ & $2(2.5 \%) 15(19.4 \%)$ & 0.622 \\
III IV & $37(34.3 \%) 46$ & $(34.7 \%) 11(43.6 \%)$ & $26(33.7 \%) 35$ \\
& $(42.5 \%)$ & $(45.4 \%)$ & \\
Treatment type & $64(59.3 \%) 44$ & $17(54.8 \%) 14$ & $47(61 \%) 30(39 \%)$ & 0.553 \\
Pazopanib Sunitinib & $(40.7 \%)$ & $(45.2 \%)$ & \\
IMDC risk group & $9(8.3 \%) 66(61.1 \%)$ & $3(9.6 \%) 19(61.2 \%)$ & $6(7.7 \%) 47(61 \%)$ & 1 \\
Favorable & $33(30.5 \%)$ & $9(29 \%)$ & $24(31.1 \%)$ & \\
Intermediate Poor & & & \\
\hline
\end{tabular}

Abbreviations: SD: Standard Deviation; PFS: Progression-Free Survival; OS: Overall Survival.

Table 2: Body composition parameters at the baseline and follow-up CT performed 3-4 months after treatment initiation.

\begin{tabular}{lllll}
\hline & All patients $(\mathrm{n}=108)$ & Female $(\mathrm{n}=31)$ & Male $(\mathrm{n}=77)$ & \\
\hline Body composition variables & Mean \pm SD & Mean \pm SD & Mean \pm SD & P value \\
Height, cm & $1.70(0.007)$ & $1.61(0.06)$ & $1.71(0.05)$ & $<0.001$ \\
Weight, $\mathrm{kg}$ & $75(12.95)$ & $70(10.57)$ & $77(13.17)$ & 0.004 \\
BMI $\left(\mathrm{kg} / \mathrm{m}^{2}\right)$ & $25.95(3.89)$ & $26.76(3.83)$ & $25.82(3.93)$ & 0.541 \\
First CT VATI, $\mathrm{cm}^{2} / \mathrm{m}^{2}$ & $47.91(30.84)$ & $57.70(28.1)$ & $44.38(31.82)$ & 0.173 \\
Second CT VATI cm $/ \mathrm{m}^{2}$ & $30.06(36.78)$ & $21.35(38.97)$ & $33.19(36.13)$ & 0.831 \\
VATI change, cm $\mathrm{cm}^{2}$ & $1.10(80.47)$ & $-1.02(50.96)$ & $1.57(89.71)$ & 0.906 \\
First CT SATI, $\mathrm{cm}^{2} / \mathrm{m}^{2}$ & $53.46(35.47)$ & $81.15(37.33)$ & $47.54(29.28)$ & $<0.001$ \\
Second CT SATI, $\mathrm{cm}^{2} / \mathrm{m}^{2}$ & $46.37(40.66)$ & $60.09(56.15)$ & $37.48(41.21)$ & 0.217 \\
SATI change, $\mathrm{cm}^{2}$ & $4.64(68.45)$ & $-8(75.03)$ & $5.3(64.66)$ & 0.140 \\
First CT TATI, $\mathrm{cm}^{2} / \mathrm{m}^{2}$ & $53.46(35.47)$ & $81.15(37.33)$ & $47.54(29.28)$ & $<0.001$ \\
Second CT TATI, $\mathrm{cm}^{2} / \mathrm{m}^{2}$ & $85.37(77.82)$ & $92.43(91.11)$ & $78.85(71.97)$ & 0.512 \\
TATI change, $\mathrm{cm}^{2} / \mathrm{m}^{2}$ & $3.10(128.47)$ & $0.38(110.78)$ & $4.75(134.39)$ & 0.516 \\
First CT SMI, $\mathrm{cm}^{2} / \mathrm{m}^{2}$ & $49.62(11.33)$ & $46.51(6.41)$ & $51.88(12.54)$ & 0.01 \\
Second CT SMI, $\mathrm{cm}^{2} / \mathrm{m}^{2}$ & $38.96(23.76)$ & $33.92(23.08)$ & $41.33(24.08)$ & 0.266 \\
SMI change, $\mathrm{cm}^{2} / \mathrm{m}^{2}$ & $-6.96(38.09)$ & $-6.96(39.17)$ & $-7.58(37.98)$ & 0.906 \\
First CT psoas index, $\mathrm{cm}^{2} / \mathrm{m}^{2}$ & $5.43(1.82)$ & $4.6(1.3)$ & $5.98(1.85)$ & $<0.001$ \\
Second CT psoas index, $\mathrm{cm}^{2} / \mathrm{m}^{2}$ & $4.1(3.03)$ & $4.05(2.53)$ & $4.3(3.19)$ & 0.245 \\
Psoas index change, $\mathrm{cm}^{2} / \mathrm{m}^{2}$ & $-1.02(4.6)$ & $-0.48(2.67)$ & $-1.37(5.13)$ & 0.129 \\
\hline
\end{tabular}

Abbreviations; SD: Standard Deviation; BMI: Body Mass Index, CT: Computerized Tomography, VATI: Visceral Adipose Tissue Index, SATI: Subcutaneous Adipose Tissue Index, TATI: total adipose tissue index, SMI: Skeletal Muscle Index.

Table 3 : Univariate Cox regression models estimating the potential influence of clinic-pathologic features and body composition variables on PFS and OS times.

\begin{tabular}{|c|c|c|c|c|}
\hline & PFS & PFS & OS & OS \\
\hline Charecteristic & HR $(95 \%$ CI $)$ & $P$ value & HR $(95 \% \mathrm{CI})$ & $P$-value \\
\hline Age, years & $\begin{array}{l}1.008 \\
(0.986-1.030)\end{array}$ & 0.464 & $\begin{array}{l}1.001 \\
(0.968-1.036)\end{array}$ & 0.937 \\
\hline $\begin{array}{l}\text { Gender Male } \\
\text { Female }\end{array}$ & $\begin{array}{l}1 \text { (reference) } 1.413 \\
(0.912-2,188)\end{array}$ & 0.121 & $\begin{array}{l}1 \text { (reference) } 1.631 \\
(0.743-3.581\end{array}$ & 0.223 \\
\hline
\end{tabular}




\begin{tabular}{|c|c|c|c|c|}
\hline & PFS & PFS & OS & OS \\
\hline $\begin{array}{l}\text { IMDC risk group } \\
\text { Favourable } \\
\text { Intermediate Poor }\end{array}$ & $\begin{array}{l}1 \text { (reference) } \\
1.251 \\
(0.589-2.655) 7.29 \\
(3.090-17.214)\end{array}$ & $0.039<0.001$ & $\begin{array}{l}1 \text { (reference) } \\
1.058 \\
(0.846-2.214) \\
8.545 \\
(4.070-18.300)\end{array}$ & $0.769<0.001$ \\
\hline $\begin{array}{l}\text { Tumor grade I II III } \\
\text { IV }\end{array}$ & $\begin{array}{l}1 \text { (reference) } 1.575 \\
(0.515-4.815) 1.522 \\
(0.529-4.378) \\
(0.566-4.678)\end{array}$ & 0.843 & $\begin{array}{l}1 \text { (reference) } 1.170 \\
(0.129-10.610) 2.314 \\
(0.3-17.859) 3.014 \\
(0.396-22.943)\end{array}$ & 0.317 \\
\hline $\operatorname{BMI}\left(\mathrm{kg} / \mathrm{m}^{2}\right)$ & $\begin{array}{l}0.955 \\
(0.903-1.009)\end{array}$ & 0.101 & $\begin{array}{l}0.944 \\
(0.846-1.041)\end{array}$ & 0.245 \\
\hline $\begin{array}{l}\text { First CT SATI, } \\
\mathrm{cm}^{2} / \mathrm{m}^{2}\end{array}$ & $1(0.993-1.006)$ & 0.885 & $\begin{array}{l}0.993 \\
(0.981-1.005)\end{array}$ & 0.145 \\
\hline $\begin{array}{l}\text { SATI change, } \\
\mathrm{cm}^{2} / \mathrm{m}^{2}\end{array}$ & $\begin{array}{l}1.001 \\
(0.998-1.005)\end{array}$ & 0.499 & $\begin{array}{l}1.002 \\
(0.995-1.008)\end{array}$ & 0.499 \\
\hline $\begin{array}{l}\text { First CT VATI, } \\
\mathrm{cm}^{2} / \mathrm{m}^{2}\end{array}$ & $\begin{array}{l}0.996 \\
(0.989-1.003)\end{array}$ & 0.240 & $\begin{array}{l}0.992 \\
(0.980-1.003)\end{array}$ & 0.158 \\
\hline $\begin{array}{l}\text { VATI change, } \\
\mathrm{cm}^{2} / \mathrm{m}^{2}\end{array}$ & $1(0.998-1.003)$ & 0.764 & $\begin{array}{l}1.002 \\
(0.996-1.008)\end{array}$ & 0.496 \\
\hline $\begin{array}{l}\text { First CT TATI, } \\
\mathrm{cm}^{2} / \mathrm{m}^{2}\end{array}$ & $1(0.993-1.006)$ & 0.885 & $\begin{array}{l}0.993 \\
(0.981-1.005)\end{array}$ & 0.145 \\
\hline $\begin{array}{l}\text { TATI change, } \\
\mathrm{cm}^{2} / \mathrm{m}^{2}\end{array}$ & $\begin{array}{l}1.001 \\
(0.999-1.002)\end{array}$ & 0.593 & $\begin{array}{l}1.001 \\
(0.997-1.005)\end{array}$ & 0.516 \\
\hline $\begin{array}{l}\text { First CT SMI, } \\
\mathrm{cm}^{2} / \mathrm{m}^{2}\end{array}$ & $\begin{array}{l}0.979 \\
(0.961-0.997)\end{array}$ & 0.021 & $\begin{array}{l}1.012 \\
(0.980-1.045)\end{array}$ & 0.477 \\
\hline SMI change & $\begin{array}{l}0.999 \\
(0.992-1.005)\end{array}$ & 0.706 & $\begin{array}{l}0.997 \\
(0.986-1.008)\end{array}$ & 0.575 \\
\hline $\begin{array}{l}\text { First CT Psoas } \\
\text { index }\end{array}$ & $\begin{array}{l}0.871(0.776- \\
0.978)\end{array}$ & 0.020 & $\begin{array}{l}1.001 \\
(0.848-1.181)\end{array}$ & 0.993 \\
\hline $\begin{array}{l}\text { Psoas index } \\
\text { change }\end{array}$ & $\begin{array}{l}0.996 \\
(0.938-1.056)\end{array}$ & 0.888 & $\begin{array}{l}0.962 \\
(0.895-1.035)\end{array}$ & 0.302 \\
\hline
\end{tabular}

Abbreviations; IMDC: International Metastatic renal cell carcinoma Database Consortium, BMI: Body Mass Index, CT: Computerized Tomography, CI: Confidence Interval, HR: Hazard Ratio, OS: Overall Survival; PFS: Progression-Free Survival, VATI: Visceral Adipose Tissue Index, SATI: Subcutaneous Adipose Tissue Index, TATI: total adipose tissue index, SMI: Skeletal Muscle Index, SMA: Skeletal Muscle Area

Table 4 : Multivariate Cox regression models analyzing the potential influence of body composition parameters on PFS and OS times.

\begin{tabular}{|c|c|c|c|c|}
\hline & HR & 95\% CI for HR & 95\% CI for $\mathrm{HR}$ & $\mathrm{P}$ value \\
\hline & & lower & Upper & \\
\hline \multicolumn{5}{|l|}{ Overall survival* } \\
\hline SATI & 0.976 & 0.957 & 0.996 & 0.019 \\
\hline VATI & 1.005 & 1.001 & 1.009 & 0.024 \\
\hline TATI & 0.982 & 0.966 & 0.999 & 0.035 \\
\hline \multicolumn{5}{|l|}{ Progression-free Survival } \\
\hline SMI & 0.975 & 0.955 & 0.995 & 0.015 \\
\hline IMDC risk group Favourable Intermediate poor & 1 (reference) 1.4977 .972 & 0.6883 .186 & 3.35619 .945 & $<0.0010$ \\
\hline
\end{tabular}


*SATI, VATI and TATI were evaluated one by one with in the multivariate analyses because of multicollinearity between these variables. In addition to these parameters, only IMDC scoring system was found as an independent predictor.

Abbreviations; HR: Hazard Ratio, VATI: Visceral Adipose Tissue Index, SATI: Subcutaneous Adipose Tissue Index, TATI: total adipose tissue index, SMI: Skeletal Muscle Index, SMA: Skeletal Muscle Area. IMDC: International Metastatic renal cell carcinoma Database Consortium 